\title{
Cutaneous Human Polyomavirus Small T Antigens and 4E-BP1 Targeting
}

\author{
Julie H. Wua, bebecca A. Simonette ${ }^{a}$ Thomas Hsiao ${ }^{a}$ Hung Q. Doan ${ }^{a}$ \\ Peter L. Rady ${ }^{\text {a Stephen K. Tyring }}{ }^{\text {a }}$ \\ ${ }^{a}$ Department of Dermatology, University of Texas Medical School at Houston, and b Baylor College of \\ Medicine, Houston, Tex., USA
}

\section{Key Words}

Human polyomavirus $\cdot$ Small T antigen $\cdot 4 \mathrm{E}$-binding protein $1 \cdot$ Merkel cell polyomavirus - Trichodysplasia spinulosa-associated polyomavirus · Human

polyomavirus $6 \cdot$ Human polyomavirus 7

\begin{abstract}
Merkel cell polyomavirus (MCPyV), trichodysplasia spinulosa-associated polyomavirus (TSPyV), human polyomavirus 6 (HPyV6), and human polyomavirus 7 (HPyV7) are implicated in the pathogeneses of distinct hyperproliferative cutaneous growths and encode small tumor (ST) antigens. The current study demonstrates that the four ST antigens differentially regulate 4E-binding protein 1 (4E-BP1) serine 65 hyperphosphorylation. MCPyV and HPyV7 ST antigens were found to promote the presence of the hyperphosphorylated 4E-BP1- $\delta$ isoform, while TSPyV and HPyV6 sT antigens had no significant effects. Given that hyperphosphorylated $4 \mathrm{E}-\mathrm{BP} 1$ is associated with an aggressive cancer phenotype, our findings confirm the previously reported pathogenicity of MCPyV ST and highlight a novel mechanism by which HPyV7 sT may mediate oncogenesis.

(c) 2016 S. Karger AG, Basel
\end{abstract}

\section{Introduction}

In 2008, the discovery of the Merkel cell polyomavirus $(\mathrm{MCPyV})$ shed light on the underlying viral pathomechanisms of Merkel cell carcinoma, a highly aggressive neuroendocrine skin cancer [1]. While $\mathrm{MCPyV}$ is, to date, the only oncogenic virus of the polyomavirus family, trichodysplasia spinulosa-associated polyomavirus (TSPyV), human polyomavirus 6 (HPyV6), and human polyomavirus 7 (HPyV7) have all been associated with hyperproliferative epithelial growths. Currently, TSPyV, HPyV6, and HPyV7 are implicated in the development and progression of trichodysplasia spinulosa, the neoplastic growth of keratinocytes in response to BRAF inhibitors, and the pathogenesis of human thymic epithelial tumors, respectively [2-5].

As with other polyomaviruses, MCPyV, TSPyV, HPyV6, and HPyV7 are double-stranded DNA viruses that encode regulatory tumor (T) antigens. Notably, polyomavirus small $\mathrm{T}(\mathrm{sT})$ antigens are strongly associated with cell proliferation pathways [6-9]. For example, in a previous report, we showed that TSPyV sT antigen activates the MEK-ERK-c-Jun pathway [7].

\section{KARGER}

E-Mail karger@karger.com www.karger.com/int
(C) 2016 S. Karger AG, Basel

0300-5526/16/0586-0382\$39.50/0
Stephen K. Tyring

6431 Fannin Street

Houston, TX 77030 (USA)

E-Mail styring@ccstexas.com 
Recently, Shuda et al. [9] demonstrated that MCPyV sT-mediated oncogenesis was largely dependent on its ability to regulate cap-dependent protein translation pathways. In particular, MCPyV sT antigen facilitated stabilization of the hyperphosphorylated $\delta$-isoform $4 \mathrm{E}$ binding protein 1 (4E-BP1), which was essential for the induction of cellular transformation [9]. In the current study, we aim to further explore the emerging roles of 4E$\mathrm{BP} 1$ in sT antigen pathomechanisms of human polyomaviruses. Our findings indicate that $4 \mathrm{E}-\mathrm{BP} 1$ is differentially regulated by the MCPyV, TSPyV, HPyV6, and HPyV7 sT antigens, which provide novel mechanistic insight into the patho-oncogenic potential of these viruses.

\section{Materials and Methods}

Cell Lines and Cell Culture

HPyV7, MCPyV, TSPyV, and HPyV6 sT antigen-coding genes were subcloned individually into lentiviral vectors that contained tetracycline-inducible CMV promoters and blasticidin antibiotic selection markers (GenTarget Inc). HEK293 cells were infected with the lentiviral constructs; expression of the sT antigen was confirmed by Western blotting with the appropriate custom mouse monoclonal antibodies (GenScript Inc). The antibodies were raised against the HPyV7 sT (clone ID 6H2G7, 1:10,000), MCPyV sT (4H5D4, 1:1000), TSPyV sT (2E10B11, 1:750), and HPyV6 sT (10B11F10, 1:250) epitopes. Cellular proteins were extracted with Mammalian Protein Extraction Reagent ${ }^{\mathrm{TM}}$ (Pierce) according to the manufacturer's protocol and lysates were prepared with Halt Protease Inhibitor Cocktail (Thermo Fisher Scientific) supplemented with phosphatase inhibitors.

Western Blotting

Cell lysates were clarified on polyacrylamide gels (10-20\% Tris-Glycine; Novex, Invitrogen-Life Technologies). Separated proteins were transferred to polyvinylidine difluoride membranes (Invitrogen-Life Technologies) and Western immunoblot was performed against total 4E-BP1 (9452) and phospho-4E-BP1 $1^{\mathrm{S} 65}$ (9456) primary antibodies (Cell Signaling Technology). GAPDH was used as the loading control (GeneTex Inc). SuperSignal West Femto chemiluminescent substrate (Thermo Fisher Scientific) was used to visualize protein bands.

\section{Results}

To delineate the roles of MCPyV, TSPyV, HPyV6, and HPyV7 sT antigens, inducible cell lines expressing the respective sT antigen proteins were established. The effects of these sT antigens on 4E-BP1 were analyzed by Western blotting with antibodies against total or phospho-sitespecific 4E-BP1 (serine 65). Our Western blot experiments confirmed that MCPyV sT antigen markedly en-

Cutaneous HPyV sT Antigens and

4E-BP1 Targeting hanced the presence of the hyperphosphorylated $4 \mathrm{E}-\mathrm{BP} 1-\delta$ isoform (fig. 1a). We observed that the HPyV7 sT antigen also promoted the $4 \mathrm{E}-\mathrm{BP} 1-\delta$ isoform through S65 phosphorylation under the same experimental conditions (fig. 1b). In contrast, neither TSPyV sT nor HPyV6 sT antigen expression was associated with the presence of the hyperphosphorylated $4 \mathrm{E}-\mathrm{BP} 1-\delta$ isoform (fig. $1 \mathrm{c}, \mathrm{d}$ ).

\section{Discussion}

4E-BP1 plays a central role in protein synthesis by regulating the assembly of the translation initiation machinery at the mRNA 5' 7 -methylguanosine GTP cap $[10,11]$. In its unphosphorylated active conformation, 4E-BP1 binds and inhibits eukaryotic initiation factor 4E (eIF4E) [10]. As uninhibited eIF4E is essential for ribosomal recruitment and subsequent translational activation of oncogenic drivers such as cyclin D1, c-myc, VEGF, and survivin, 4E-BP1 sequestration of eIF4E represents an important step that negatively regulates protein synthesis and represses oncogenesis [10-12]. In the current study, we verified that $\mathrm{MCPyV}$ sT antigen does indeed induce 4EBP1 hyperphosphorylation. Additionally, our data implicate HPyV7 (but not HPyV6 nor TSPyV) sT antigen in a similar mechanism involving 4E-BP1 deactivation. These findings are noteworthy, given that hyperphosphorylation of 4E-BP1, especially on the $\mathrm{S} 65$ residue, is known to functionally disable its eIF4E-binding ability and thus increase protein synthesis and mitogenesis $[11,13]$.

There are four isoforms of $4 \mathrm{E}-\mathrm{BP} 1(\alpha, \beta, \gamma$, and $\delta)$, each of which is known to be sequentially phosphorylated [11, $14]$. Among these isoforms, $4 \mathrm{E}-\mathrm{BP} 1-\delta$ attracts considerable attention, as this isoform is present at its highest level during mitosis [13]. The current study is the first to demonstrate that HPyV7 sT antigen promotes the presence of the hyperphosphorylated $4 \mathrm{E}-\mathrm{BP} 1-\delta$ isoform. This finding suggests a novel mechanism of 4E-BP1 in HPyV7 pathogenesis and provides evidence supporting a possible role for the virus in neoplastic cell growth. Although HPyV7 infection has been associated with epithelial thymomas and pruritic dermal rash, its specific roles in cutaneous diseases remain largely undefined $[4,5]$. In this context, it is notable that $4 \mathrm{E}-\mathrm{BP} 1$ hyperphosphorylation correlates with aggressive cancer growth and worse prognosis for metastatic melanoma patients $[9,15,16]$. Additionally, previous studies have shown that $4 \mathrm{E}-\mathrm{BP} 1$ phosphorylation status can serve as a predictive marker for drug resistance and a proangiogenic phenotype $[16,17]$. As $4 \mathrm{E}-\mathrm{BP} 1$ is a downstream target of the Akt/mTORC1 


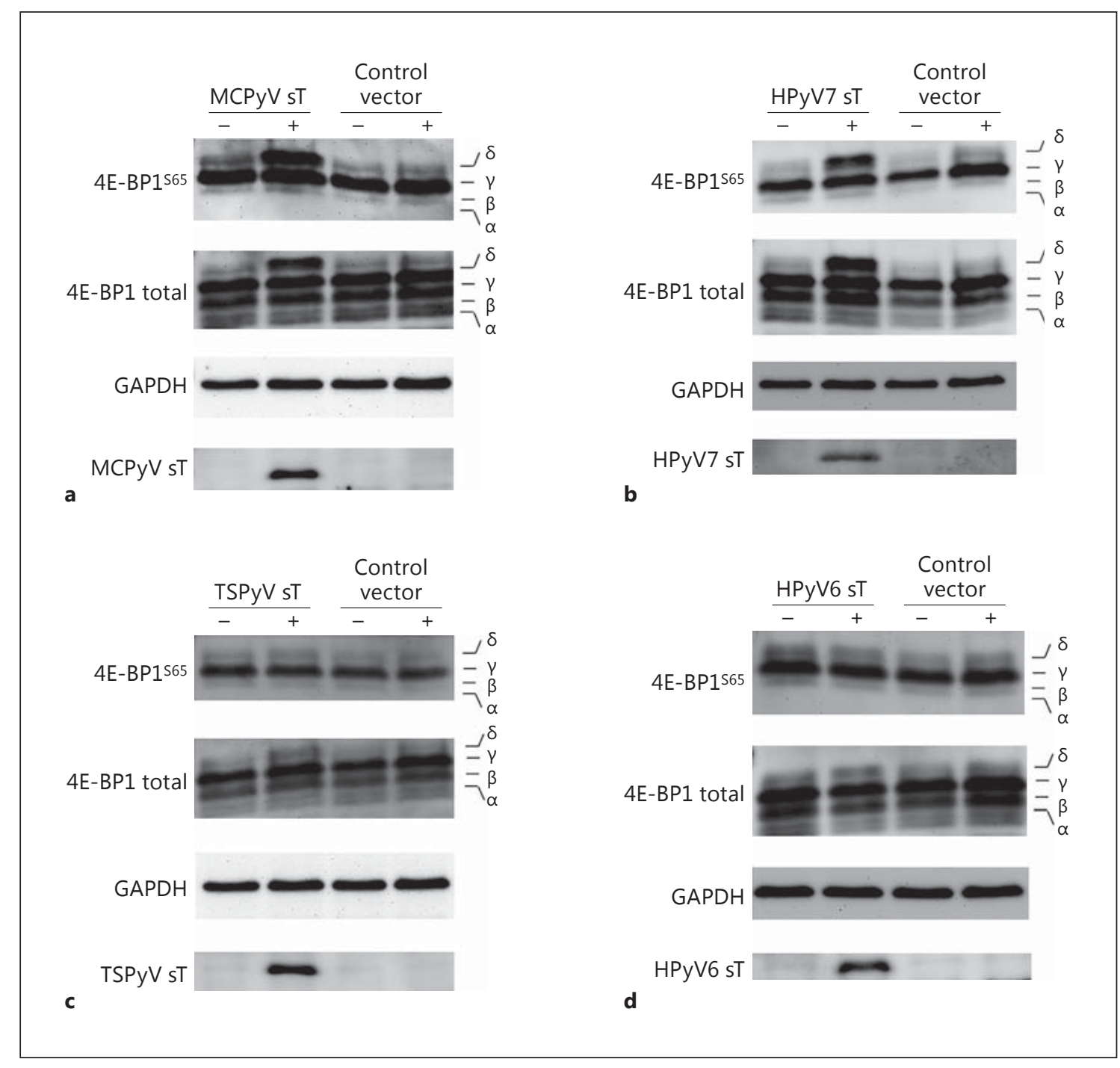

Fig. 1. MCPyV, TSPyV, HPyV6, and HPyV7 differentially target $4 \mathrm{E}-\mathrm{BP} 1$. Cells were transduced with lentiviral vectors containing specific sT-coding genes or the control empty lentiviral vector. Cellular proteins were subjected to SDS-PAGE and Western blot analysis to evaluate 4E-BP1 phosphorylation status. Lanes designated with a plus sign $(+)$ contained lysates from cells induced to express the appropriate coding gene; lanes designated with a mi-

pathway, it would be logical to consider that polyomavirus sT antigens modulate this pathway due to their interactions with $4 \mathrm{E}-\mathrm{BP} 1$. However, it has been shown that MCPyV sT antigen-mediated 4E-BP1 hyperphosphorylation is largely independent of this pathway [9]. Interestingly, Shuda et al. [13] reported that $4 \mathrm{E}-\mathrm{BP} 1-\delta$ is targeted by the CDK1/CYCB complex, which is activated upon $\mathrm{MCPyV}$ sT antigen expression. Given the findings of our current study, further investigation is needed to clarify nus sign (-) contained lysates from noninduced cells. Formation of the hyperphosphorylated mitosis-specific $\delta$ isoform of $4 \mathrm{E}-\mathrm{BP} 1$ was markedly elevated by expression of MCPyV sT (a) and HPyV7 sT (b). In contrast to MCPyV and HPyV7, the presence of TSPyV sT (c) and HPyV6 sT (d) did not result in significant change of the $4 \mathrm{E}-\mathrm{BP} 1$ phosphorylation status or the manifestation of the $\delta$ isoform.

the role of HPyV7 sT antigen in modulating 4E-BP1 hyperphosphorylation. Whether HPyV7 sT-associated 4EBP1 deactivation occurs through a similar or differential mechanism compared to MCPyV sT antigen remains unknown. As Merkel cell carcinoma is more aggressive than HPyV7-associated epithelial proliferations, it is possible that these two sT antigens mediate hyperphosphorylation of 4E-BP1 through separate but parallel pathways. Investigating this differential activation of 4E-BP1 hyperphos- 
phorylation would shed light on disparate pathway interactions that mediate an aggressive cancer phenotype.

The pathobiological effects and mechanisms of polyomavirus sT antigens are complex and dependent on the characteristics of different viruses as well as the specific tissue/cell context $[7,8,18]$. Computer-assisted genomic analysis of MCPyV, TSPyV, HPyV6, and HPyV7 demonstrated that all four sT antigens share an $\mathrm{N}$-terminal DnaJ domain followed by protein phosphatase $2 \mathrm{~A}$-binding sequences and zinc-finger motifs $[19,20]$. Further sT truncation studies are required to determine whether these attributes in $\mathrm{HPyV} 7 \mathrm{sT}$ contribute to $4 \mathrm{E}-\mathrm{BP} 1$ hyperphosphorylation.
In summary, the current study emphasizes the differential effects of MCPyV, TSPyV, HPyV6, and HPyV7 sT antigens on 4E-BP1 phosphorylation. Our results demonstrate that $4 \mathrm{E}-\mathrm{BP} 1-\delta$ may represent a common target of both HPyV7 and MCPyV sT antigens; as 4E-BP1 hyperphosphorylation and $\delta$ isoform expression are associated with aggressive cutaneous disease, this finding highlights a novel pathway that implicates HPyV7 in oncogenesis. At present, 4E-BP1 phosphorylation has not been implicated in the actions of any other polyomavirus sT antigens. Further studies are warranted to determine whether $4 \mathrm{E}-\mathrm{BP} 1$ can be targeted for the treatment of $\mathrm{HPyV} 7$ - and MCPyV-associated diseases.

\section{References}

1 Feng H, Shuda M, Chang Y, Moore PS: Clonal integration of a polyomavirus in human Merkel cell carcinoma. Science 2008;319: 1096-1100.

2 van der Meijden E, Janssens RW, Lauber C, Bouwes Bavinck JN, Gorbalenya AE, Feltkamp MC: Discovery of a new human polyomavirus associated with trichodysplasia spinulosa in an immunocompromized patient. PLoS Pathog 2010;6:e1001024.

3 Schrama D, Groesser L, Ugurel S, Hafner C, Pastrana DV, Buck CB, Cerroni L, Theiler A, Becker JC: Presence of human polyomavirus 6 in mutation-specific BRAF inhibitor-induced epithelial proliferations. JAMA Dermatol 2014;150:1180-1186.

4 Rennspiess D, Pujari S, Keijzers M, AbdulHamid MA, Hochstenbag M, Dingemans AM, Kurz AK, Speel EJ, Haugg A, Pastrana DV, Buck CB, De Baets MH, Zur Hausen A: Detection of human polyomavirus 7 in human thymic epithelial tumors. J Thorac Oncol 2015; 10:360-366.

5 Ho J, Jedrych JJ, Feng H, Natalie AA, Grandinetti L, Mirvish E, Crespo MM, Yadav D, Fasanella KE, Proksell S, Kuan SF, Pastrana DV, Buck CB, Shuda Y, Moore PS, Chang Y: Human polyomavirus 7 -associated pruritic rash and viremia in transplant recipients. J Infect Dis 2015;211:1560-1565.

6 Nguyen HP, Patel A, Simonette RA, Rady P, Tyring SK: Binding of the trichodysplasia spinulosa-associated polyomavirus small $\mathrm{T}$ antigen to protein phosphatase 2A: elucidation of a potential pathogenic mechanism in a rare skin disease. JAMA Dermatol 2014;150: 1234-1236.
$7 \mathrm{Wu}$ JH, Simonette RA, Nguyen HP, Rady PL, Tyring SK: Small T-antigen of the TS-associated polyomavirus activates factors implicated in the MAPK pathway. J Eur Acad Dermatol Venereol 2015, doi: 10.1111/jdv.13111.

8 Kwun HJ, Shuda M, Camacho CJ, Gamper AM, Thant M, Chang Y, Moore PS: Restricted protein phosphatase 2A targeting by Merkel cell polyomavirus small $\mathrm{T}$ antigen. J Virol 2015;89:4191-4200.

9 Shuda M, Kwun HJ, Feng H, Chang Y, Moore PS: Human Merkel cell polyomavirus small T antigen is an oncoprotein targeting the $4 \mathrm{E}$ BP1 translation regulator. J Clin Invest 2011; 121:3623-3634.

10 Richter JD, Sonenberg N: Regulation of capdependent translation by eIF4E inhibitory proteins. Nature 2005;433:477-480.

11 Karim MM, Hughes JM, Warwicker J, Scheper GC, Proud CG, McCarthy JE: A quantitative molecular model for modulation of mammalian translation by the eIF4E-binding protein 1. J Biol Chem 2001;276:2075020757.

12 Mamane Y, Petroulakis E, Rong L, Yoshida K, Ler LW, Sonenberg N: eIF4E - from translation to transformation. Oncogene 2004;23: 3172-3179.

13 Shuda M, Velasquez C, Cheng E, Cordek DG, Kwun HJ, Chang Y, Moore PS: CDK1 substitutes for mTOR kinase to activate mitotic capdependent protein translation. Proc Natl Acad Sci USA 2015;112:5875-5882.
14 Gingras AC, Raught B, Gygi SP, Niedzwiecka A, Miron M, Burley SK, Polakiewicz RD, Wyslouch-Cieszynska A, Aebersold R, Sonenberg N: Hierarchical phosphorylation of the translation inhibitor 4E-BP1. Genes Dev 2001;15:2852-2864.

15 O'Reilly KE, Warycha M, Davies MA, Rodrik V, Zhou XK, Yee H, Polsky D, Pavlick AC, Rosen N, Bhardwaj N, Mills G, Osman I: Phosphorylated 4E-BP1 is associated with poor survival in melanoma. Clin Cancer Res 2009; 15:2872-2878.

16 Korkolopoulou P, Levidou G, El-Habr EA, Piperi C, Adamopoulos C, Samaras V, Boviatsis E, Thymara I, Trigka EA, Sakellariou S, Kavantzas N, Patsouris E, Saetta AA: Phosphorylated $4 \mathrm{E}$-binding protein 1 ( $\mathrm{p}-4 \mathrm{E}-\mathrm{BP} 1)$ : a novel prognostic marker in human astrocytomas. Histopathology 2012;61:293-305.

17 Zhang Y, Zheng XF: mTOR-independent 4E$\mathrm{BP} 1$ phosphorylation is associated with cancer resistance to mTOR kinase inhibitors. Cell Cycle 2012;11:594-603.

18 Rodriguez-Viciana P, Collins C, Fried M Polyoma and SV40 proteins differentially regulate PP2A to activate distinct cellular signaling pathways involved in growth control. Proc Natl Acad Sci USA 2006;103:1929019295.

19 Van Ghelue M, Khan MT, Ehlers B, Moens U: Genome analysis of the new human polyomaviruses. Rev Med Virol 2012;22:354-377.

20 Spurgeon ME, Lambert PF: Merkel cell polyomavirus: a newly discovered human virus with oncogenic potential. Virology 2013;435: 118-130. 